\title{
Comparison of growth and pubertal progression in wild type female rats with different bedding types
}

\author{
Byung Ho Kang, MD', \\ Shin-Hee Kim, MD', \\ Kyung A Jung, $M D^{2}$, \\ So Youn Kim, MD', \\ Sung-Hoon Chung, MD', \\ Young Shil Park, MD', \\ Kyung Lim Yoon, MD', \\ Kye Shik Shim, MD ${ }^{1}$
}

'Department of Pediatrics, Kyung Hee University Hospital at Gangdong, Seoul, 'Department of Pediatrics, Kyung Hee University Hospital, Kyung Hee University School of Medicine, Seoul, Korea
Purpose: Endocrine-disrupting chemicals interfere with the endocrine system and therefore affect growth and pubertal progression. The study aim was to compare the growth and pubertal progression in wild-type female rats with different bedding types.

Methods: Twenty 5-week-old female wild-type Sprague Dawley rats were randomly assigned to two groups with different bedding types: one group received wood shaving bedding, while a second group received corncob bedding. We determined crown-rump length and body weight as anthropometric measurements and assessed the serum growth hormone $(\mathrm{GH})$ and estradiol levels. The gh1 mRNA expression levels were compared using quantitative real time transcription polymerase chain reaction. The estrous cycle was evaluated by vaginal smear.

Results: The anthropometric measurements were not significantly different between the two groups. The mean relative expression of the gh1 gene was lower in the corncob bedding group than that in the wood shaving group $(P=0.768)$. Meanwhile serum GH and estradiol were increased in the wood shaving bedding group; however this difference was not statistically significant. The time to first estrus and the length of the estrous cycle were increased in the corncob bedding group; the proportion of normal estrous cycles was also decreased. These findings indicate irregularities in the estrous cycle.

Conclusion: Endocrine-disrupting chemicals in corncob bedding might be associated with time to first estrus and length of the estrous cycle. Therefore, the type of bedding should be considered as a factor affecting pubertal progression in rodents.

Keywords: Endocrine disrupting chemicals, Bedding, Growth, Puberty

\section{Introduction}

Endocrine-disrupting chemicals are exogenous agents that interfere with the synthesis, secretion, transport, metabolism, binding action, or elimination of natural blood-borne hormones present in the body. They are responsible for homeostasis, reproduction, and developmental processes ${ }^{1)}$. They can act centrally through the hypothalamic-pituitary-gonadal axis or directly through peripheral interaction in the tissues targeted by sex steroids, modifying sexual development and function ${ }^{2)}$. Recently, many experimental animal studies have demonstrated that exposure to endocrine-disrupting agents affect growth pattern, onset, and progression of puberty. Studies have also reported the presence of a chemical agent in corncob animal bedding ${ }^{3)}$ that caused, several adverse effects, such as reduced reproductive behavior ${ }^{4}$, altered estrous cycle ${ }^{5)}$, and decreased slow-wave sleep ${ }^{6)}$.

The sign of pubertal onset in female rats is vaginal opening, which occurs about 5-9 weeks after birth. The reproductive cycle in rodents is known as the estrous cycle, which has a duration of 4-5 days over 4 stages: estrus, proestrus, metestrus, and diestrus, which can be

\author{
Received: 2 January, 2015 \\ Accepted: 25 March, 2015 \\ Address for correspondence: \\ Kye Shik Shim, MD \\ Department of Pediatrics, \\ Kyung Hee University Hospital \\ at Gangdong, 892 Dongnam-ro, \\ Gangdong-gu, Seoul 134-727, Korea \\ Tel: +82-2-440-6131 \\ Fax: +82-2-440-6073 \\ E-mail: ksshim64@gmail.com
}


confirmed by vaginal smear ${ }^{7}$.

The pubertal growth spurt results from increased synthesis and secretion of growth hormones (GHs) and insulin-like growth factor, regulated by gonadal steroids, of which estrogens are the most important ${ }^{8)}$. Estradiol is a major estrogen that affects endocrine, metabolic function, and pubertal growth spurt ${ }^{9}$.

The purpose of this study was to evaluate the effect of corncob bedding on growth and pubertal onset or progression by analyzing anthropometric measurements, serum levels of GH and estradiol, onset of vaginal openings, and changes of the estrous cycles in rats.

\section{Materials and methods}

\section{Animals}

Twenty female wild Sprague Dawley rats with an age of 4 weeks were acclimated to our laboratory for 1 week. They were divided into two groups with different bedding types. The first group received wood shaving bedding and second group, corncob bedding. The animals were housed in an approved animal facility with a 12-hour light cycle and given ad libitum access to food and water.

\section{Experiment}

1) Anthropometric measurement

Crown-rump length and body weight of the Sprague Dawley rats were measured weekly, starting a week before the application of each type of bedding

\section{2) Hormonal assays}

Blood samples were taken from the tail before and after application of the bedding. Commercially available enzymelinked immunosorbent assay kits were used to measure serum levels of GH (Millipore, cat.no.EZRMGH-45K, Darmstadt, Germany) and estradiol (CALBIOTECH, cat.no. ES180S-100, Spring valley, CA, USA).

3) Quantitative real-time PCR analysis

Expression levels of $g h 1 \mathrm{mRNA}$ in the pituitary gland were measured using quantitative real-time polymerase chain reaction (qRT-PCR) after each experiment. Total RNA was prepared using the RNeasy mini kit (Qiagen cat.no. 74104)

Table 1. Serum hormone levels before application of bedding in each group (at an age of 5 weeks)

\begin{tabular}{cccc}
\hline Hormone & Wood shaving bedding & Corncob bedding & $P$-value \\
\hline $\begin{array}{c}\text { Serum GH } \\
(\mathrm{ng} / \mathrm{mL})\end{array}$ & $4.6 \pm 2.0$ & $4.2 \pm 0.5$ & 0.778 \\
$\begin{array}{c}\text { Serum estradiol } \\
(\mathrm{pg} / \mathrm{mL})\end{array}$ & $5.4 \pm 1.2$ & $5.2 \pm 1.7$ & 0.934 \\
\hline
\end{tabular}

Values are presented as mean \pm standard deviation.

$\mathrm{GH}$, growth hormone. according to the manufacturers' instructions. RT-PCR was performed using ABI Power SYBR Green PCR Master Mix (part No. 436759) and the ABI Step One Plus Real Time PCR System.

4) Measurement of sexual maturation in rats by vaginal smear Each group was checked daily for the first day of vaginal opening. Vaginal smears were collected to determine the estrous cycle after vaginal opening. The interval between vaginal opening and the first normal estrous cycle was recorded to determine sexual maturation. Cytology was evaluated and the stage of the cycle was determined. The phases of a normal estrous cycle are proestrus, estrus, metestrus, and diestrus. We compared the proportion of normal estrous cycles in each group by measuring the number of days in each stage of the cycle, and cycle length.

\section{Statistical analysis}

Statistical analysis was performed using IBM SPSS Statistics ver. 19.0 (IBM Co., Armonk, NY, USA). The continuous variables between two groups were compared using Wilcoxon rank sum test. The numbers of normal estrous cycles between two groups
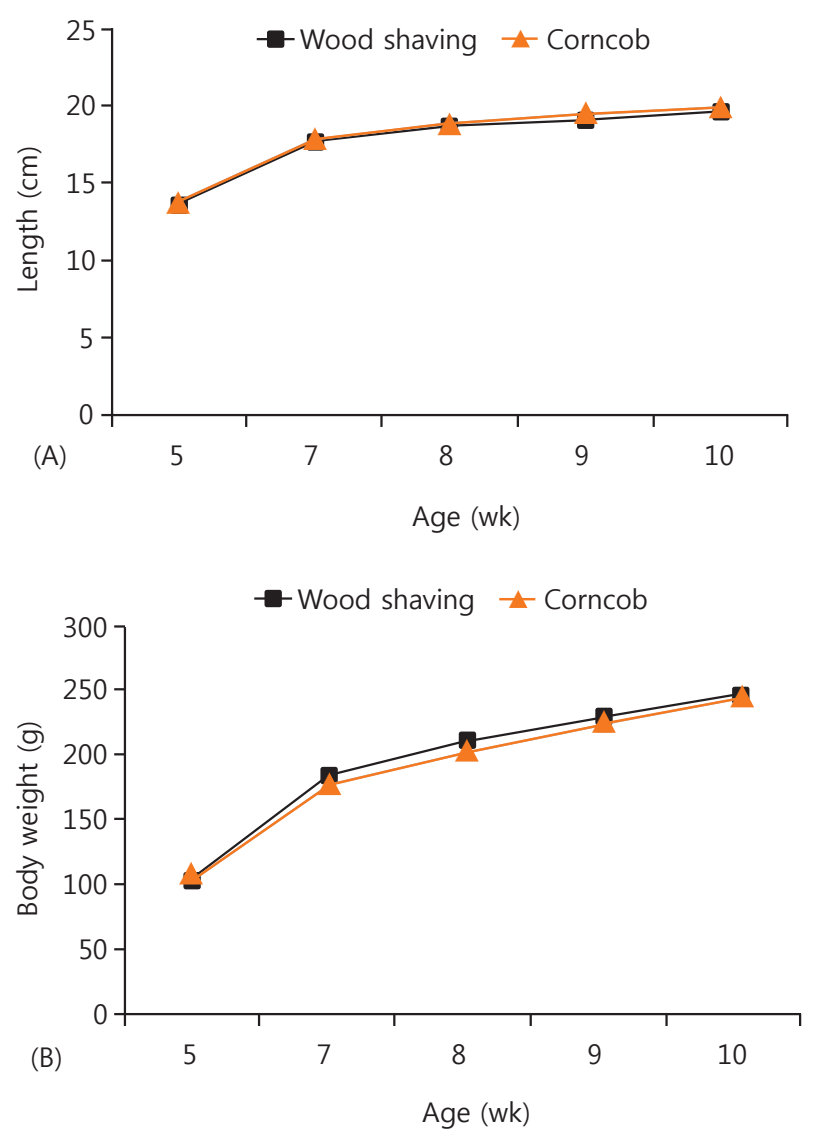

Fig. 1. Comparison of crown-rump length (A) and body weight (B) between the two groups did not show any significant differences. 
were compared using Fisher exact test. Statistical significance was defined as $P<0.05$. Results are described as mean \pm standard deviation.

\section{Results}

Anthropometric parameters were measured weekly for the two groups between the ages of 5 and 10 weeks. There were no significant differences in body length or weight between the groups before and after the application of each type of bedding $(P=0.693$ and $P=0.392)$ (Fig. 1$)$.

Serum GH and estradiol levels were not different in each group $(P=0.685$ and $P=0.809$ ) (Tables 1,2$)$. The mean level of relative expression of the $g h 1$ gene was 0.914 times lower in the corncob bedding group than in the wood shaving group, but the difference was not statistically significant $(P=0.768)$ (Fig. 2).

The mean period between vaginal opening and the first estrous cycle was $4.1 \pm 0.3$ days in the wood shaving group and $4.8 \pm 0.3$ days in the corncob bedding. The first estrus phase was significantly delayed in the corncob bedding group $(P<0.05)$ (Fig. 3). The difference in the proportion of normal estrous cycles in the wood shaving group $(83 \%)$ and the corncob bedding

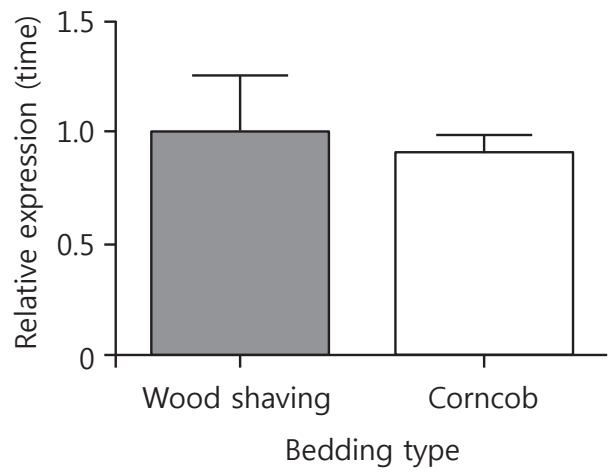

gh1 gene primer

\begin{tabular}{|c|c|c|}
\hline \multirow{2}{*}{$\begin{array}{l}\text { Oligonucleotide } \\
\text { name }\end{array}$} & \multicolumn{2}{|c|}{ Primer sequence } \\
\hline & $\begin{array}{l}\text { Sense primer } \\
\left(\text { forward, } 5^{\prime}-3^{\prime}\right)\end{array}$ & $\begin{array}{c}\text { Antisense primer } \\
\left.\text { (reverse, } 3^{\prime}-5^{\prime}\right)\end{array}$ \\
\hline Gh1 & ctt cgc ttc tcg ctg ct & gat gec ctc ttc cag gtc \\
\hline gapdh & cta ctg gcg tct tca cca c & gtt cac acc cat cac aaa ca \\
\hline
\end{tabular}

Fig. 2. Relative expression levels of gh1 gene in wood shaving and corncob bedding were measured by using real-time polymerase chain reaction. Expression of $g h 1$ in the corncob bedding group was reduced by 0.914 times compared to the wood shaving group.

Table 2. Serum hormone levels after the application of bedding for five weeks in each group (at an age of 10 weeks)

\begin{tabular}{lccc}
\hline Hormone & Wood shaving bedding & Corncob bedding & $P$-value \\
\hline $\begin{array}{c}\text { Serum } \mathrm{GH} \\
\text { (ng/mL) }\end{array}$ & $10.4 \pm 2.5$ & $9.3 \pm 1.0$ & 0.685 \\
$\begin{array}{c}\text { Serum estradiol } \\
\text { (pg/mL) }\end{array}$ & $9.1 \pm 2.3$ & $8.4 \pm 1.3$ & 0.809 \\
\hline
\end{tabular}

Values are presented as mean \pm standard deviation.

$\mathrm{GH}$, growth hormone. group (52\%) was significant $(P<0.05)$ (Fig. 4$)$. The mean estrous cycle length in the corncob bedding group was higher than in the wood shaving bedding group ( $5.5 \pm 0.3$ days compared with $4.4 \pm 0.1$ days, $P<0.05$ ) (Fig. 5). When the phases of estrous cycle over 30 days were assessed, the number of days of proestrus and estrus was significantly lower, but the length of diestrus was higher in the corncob bedding group (Fig. 6). This means that there were more irregularities in the estrous cycles in rats raised on corncob bedding than on wood shavings.

\section{Discussion}

Endocrine-disrupting chemicals can be divided into phytoestrogens, i.e., plant-derived chemical materials, and xenoestrogens, i.e., artificially created materials ${ }^{1)}$. According to their structure, phytoestrogens are classified into chromene derivatives, flavonoids, isoflavonoids, isocoumarines, chalcones, coumestans, stilbenes, lignans, ginsenosides, tetrahydrofurandiols, cinnamic acid derivatives, 2-arylbenzofurans, and phenolic compounds ${ }^{10)}$. Xenoestrogens include dic hlorodiphenyltricholoroethane and its metabolites, bisphenols,

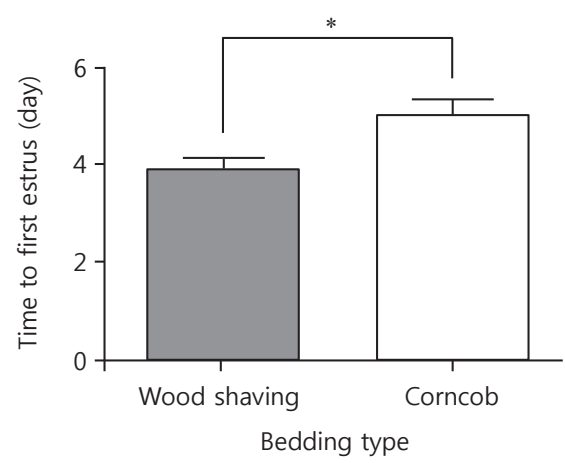

Fig. 3. Time to first estrus in each bedding type. The time was significantly delayed in corncob bedding group $(P=0.0249)$.

" $P<0.05$.

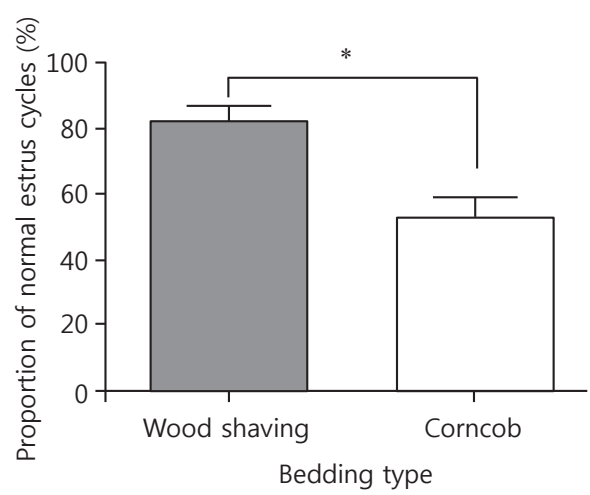

Fig. 4. Proportion of normal estrus cycles over 3 weeks. The proportion was significantly decreased in corncob bedding group $(P=0.0024)$. ${ }^{*} P<0.05$. 


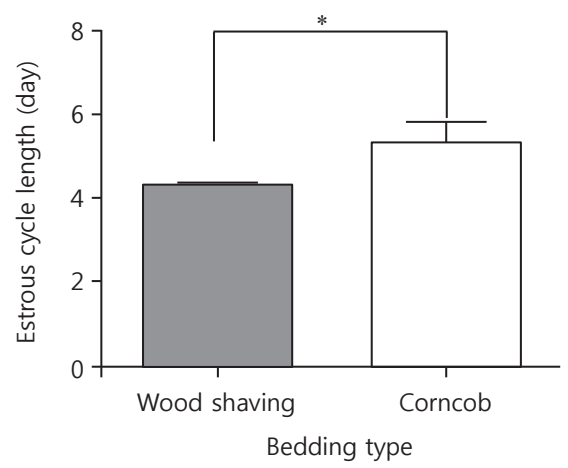

Fig. 5. Estrous cycle length of each bedding type during 3 weeks. The cycle was significantly increased in corncob bedding group $(P=0.0062)$. ${ }^{*} P<0.05$.

alkylphenols, dichlorophenols, methoxychlor, chlordecone, polychlorinated biphenyls, and dioxins ${ }^{1)}$.

Most phytoestrogens have estrogenic or antiestrogenic effects, and are connected to the regulation of reproductive organization development and cell differentiation by competitively binding to the estrogen receptor (ER) within the body ${ }^{1,11}$.

Many animal studies have been reported phytoestrogenrelated changes in puberty, delay of the estrous cycle, ovarian disorders, and changes in the function of the hypothalamus and pituitary gland. Kouki et al. ${ }^{12)}$ reported that genistein, one of the isoflavanoids present in many beans, causes early vaginal opening and irregularity of the estrous cycle. Similarly, Takagi et al. ${ }^{13)}$ founded that genistein caused irregular estrous cycling with an extended estrus. However, our results did not indicate early vaginal opening. Other studies have reported that genistein increases ovulation in low concentration through an estrogenic effect, and reduces ovulation at high concentrations through an antiestrogenic effect. This indicates the estrogenic or antiestrogenic effects are concentration-dependent ${ }^{14,15)}$.

Tetrahydrofurandiols (THF-diols) and leukotoxindiols (LTX-diols), types of phytoestrogens, originated from corncob bedding, which is frequently used as rodent bedding. Their mitogenic activity stems from an isomeric mixture of linoleic acid derivatives with a tetrahydrofuran ring and two hydroxyl groups that include 9,(12)-oxy-10,13-dihydroxystearic acid and 10,(13)-oxy-9,12-dihydroxystearic acid. These compounds apparently do not interact via classical ER pathways. Although classical $\left[{ }^{3} \mathrm{H}\right]$ estradiol exchange assays do not discriminate between ligand binding to ERa or ER $\beta$ in rat uterine nuclear fractions, the failure of the THF-diols to compete for [3H] estradiol binding in these assays suggests they do not bind to either form of the protein4). Markaverich et al ${ }^{16)}$ reported the isolation and purification of THF-diols and LTX-diols from corncob bedding by high performance liquid chromatography. THF-diols dramatically decreased sexual behavior in both male and female mice when applied in high concentrations in drinking water ( $\geq 2 \mathrm{ppm}$ ) over 30 days. LTX-diols also reduced sexual behavior of female mice when applied in high

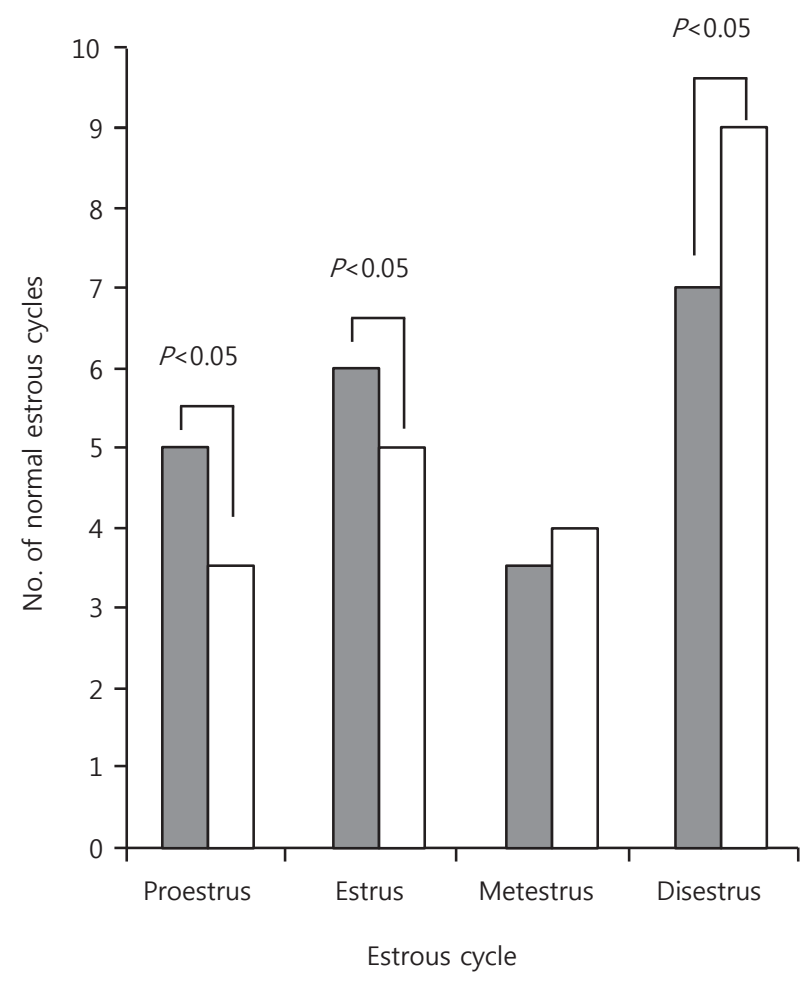

\begin{tabular}{lccr}
\hline & Wood shaving bedding & Corncob bedding & $P$-value \\
\hline Proestrus & 5.1 & 3.8 & 0.008 \\
Estrus & 6.0 & 5.0 & 0.042 \\
Metestrus & 3.7 & 4.0 & 0.969 \\
Diestrus & 7.1 & 9.0 & 0.035 \\
\hline
\end{tabular}

Fig. 6. The mean number of normal estrous cycles (proestrus, estrus, metestrus, diestrus) over 3 weeks. The mean numbers of proestrus and estrus phases were decreased $(P=0.008$ and $P=0.042)$, and that of diestrus was increased in corncob bedding group significantly $(P=0.035)$.

concentrations in drinking water ( $\geq 2 \mathrm{ppm})$. However, there was no influence on sexual behavior of male mice. Also, other studies have reported that corncob bedding reduces aggressive behavior by modifying the effect of estrogen and, decreasing expression of ERa in the brain ${ }^{17)}$. These substances can be also easily found in corn tortillas, indicating that humans may also be exposed. Previous studies are frequently performed in rats and mice because the normal rodent estrous cycle is short and regular, with a typical duration of 4-5 days, including estrus for $1-2$ days and diestrus for $2-3$ days ${ }^{17,18)}$.

This study was conducted in rats and aimed to determine the influence of endocrine-disrupting agents from corncob bedding on growth and pubertal progress. The phases of the estrous cycle were examined over 30 days via a vaginal smear. The interval between vaginal opening and the first normal estrous cycle, the proportion of normal estrous cycles, and the length of each cycle length in the two groups were also measured. Vaginal opening of rat occurs normally 5-9 weeks after birth and the first estrus phase occur during this period. Our results show that the estrous cycle was extended to an average of 5.5 days in 
the corncob bedding compared to an average of 4.4 days in the wood shaving group. There was significant difference in the time of vaginal opening and the first estrus between the two groups in our study. And, the proportion of normal estrous cycles significantly decreased in the corncob bedding group compared to the wood shaving group. We suggest that the abnormalities in the estrous cycle were caused by endocrine-disrupting agents.

In a normal pubertal growth spurt, estradiol, a gonadal steroid stimulates GH secretion until maturity is reduced ${ }^{19,20)}$. The levels of estradiol and GH in the corncob bedding group were reduced compared to the wood shaving group, in previous studies. However, this difference was not statistical significance in our study.

This study compared the influence of endocrine-disrupting agents from different bedding types on growth and pubertal progression in female rats. We found that estrous cycle irregularity is caused by agents secreted from corncob bedding. Therefore, the type of bedding is a factor that may influence puberty in rodents.

Our results on the influence of endocrine-disrupting agents on pubertal progression have several limitations. The critical window, a vulnerable to endocrine-disrupting agents, was not clearly identified. The time between exposure to endocrinedisrupting agents and the observation of a response in the body was not determined ${ }^{21)}$. The types of endocrine-disrupting agents in corncob bedding vary and may act through various mechanisms and have complex consequences in the body.

Therefore, further studies over the entire developmental period from the prenatal period to the postpubertal phase on the mechanisms by which endocrine-disrupting agents cause pubertal disorders and other possible disturbing factors in corncob bedding are necessary.

\section{Conflict of interest}

No potential conflict of interest relevant to this article was reported.

\section{References}

1. Diamanti-Kandarakis E, Bourguignon JP, Giudice LC, Hauser R, Prins GS, Soto AM, et al. Endocrine-disrupting chemicals: an Endocrine Society scientific statement. Endocr Rev 2009;30:293-342.

2. Kim SH, Park MJ. Endocrine disrupting chemicals and pubertal development. Endocrinol Metab 2012;27:20-7.

3. Krohn TC, Hansen AJ. Evaluation of corncob as bedding for rodents. Scan J Lab Anim Sci 2008;35:231-6.

4. Mani SK, Reyna AM, Alejandro MA, Crowley J, Markaverich BM. Disruption of malesexual behavior in rats by tetrahydrofurandiols (THF-diols). Steroids 2005;70:750-4.

5. Markaverich BM, Alejandro M, Thompson T, Mani S, Reyna A, Portillo W, et al. Tetrahydrofurandiols (THF-diols), leukotoxindiols (LTX-diols), and endocrine disruption in rats. Environ Health Perspect 2007;115:702-8.
6. Leys LJ, McGaraughty S, Radek RJ. Rats housed on corncob bedding show less slow-wave sleep. J Am Assoc Lab Anim Sci 2012;51:764-8.

7. Marcondes FK, Bianchi FJ, Tanno AP. Determination of the estrous cycle phases of rats: some helpful considerations. Braz J Biol 2002;62(4A):609-14.

8. Leung KC, Johannsson G, Leong GM, Ho KK. Estrogen regulation of growth hormone action. Endocr Rev 2004;25:693-721.

9. Börjesson AE, Lagerquist MK, Liu C, Shao R, Windahl SH, Karlsson C, et al. The role of estrogen receptor $\alpha$ in growth plate cartilage for longitudinal bone growth. J Bone Miner Res 2010;25:2690-700.

10. Lorand T, Vigh E, Garai J. Hormonal action of plant derived and anthropogenic non-steroidal estrogenic compounds: phytoestrogens and xenoestrogens. Curr Med Chem 2010;17:3542-74.

11. Adlercreutz H, Mazur W. Phyto-oestrogens and Western diseases. Ann Med 1997;29:95-120.

12. Kouki T, Kishitake M, Okamoto M, Oosuka I, Takebe M, Yamanouchi K. Effects of neonatal treatment with phytoestrogens, genistein and daidzein, on sex difference in female rat brain function: estrous cycle and lordosis. Horm Behav 2003;44:140-5.

13. Takagi H, Shibutani M, Lee KY, Lee HC, Nishihara M, Uneyama C, et al. Lack of modifying effects of genistein on disruption of the reproductive system by perinatal dietary exposure to ethinylestradiol in rats. Reprod Toxicol 2004;18:687-700.

14. Jefferson WN, Couse JF, Padilla-Banks E, Korach KS, Newbold RR. Neonatal exposure to genistein induces estrogen receptor (ER)alpha expression and multioocyte follicles in the maturing mouse ovary: evidence for ERbeta-mediated and nonestrogenic actions. Biol Reprod 2002;67:1285-96.

15. Jefferson WN, Padilla-Banks E, Newbold RR. Adverse effects on female development and reproduction in CD-1 mice following neonatal exposure to the phytoestrogen genistein at environmentally relevant doses. Biol Reprod 2005;73:798-806.

16. Markaverich BM, Alejandro MA, Markaverich D, Zitzow L, Casajuna N, Camarao N, et al. Identification of an endocrine disrupting agent from corn with mitogenic activity. Biochem Biophys Res Commun 2002;291:692-700.

17. Villalon Landeros R, Morisseau C, Yoo HJ, Fu SH, Hammock BD, Trainor BC. Corncob bedding alters the effects of estrogens on aggressive behavior and reduces estrogen receptor- $\alpha$ expression in the brain. Endocrinology 2012;153:949-53.

18. Goldman JM, Murr AS, Cooper RL. The rodent estrous cycle: characterization of vaginal cytology and its utility in toxicological studies. Birth Defects Res B Dev Reprod Toxicol 2007;80:84-97.

19. Freeman ME. Knobil E, Neil JD. Neuroendocrine control of the ovarian cycle of the rat. In: Knobil E, Neill JD. The 
physiology of reproduction. New York: Raven Press; 1994:613-58.

20. Hiney JK, Ojeda SR, Dees WL. Insulin-like growth factor I: a possible metabolic signal involved in the regulation of female puberty. Neuroendocrinology 1991;54:420-3.
21. Rasier G, Toppari J, Parent AS, Bourguignon JP. Female sexual maturation and reproduction after prepubertal exposure to estrogens and endocrine disrupting chemicals: a review of rodent and human data. Mol Cell Endocrinol 2006;254-255:187-201. 